\title{
Measurement of the Efficiency of Parallel Genetic Algorithm for Compress and Decompression of Fractal Imaging Using Multiple Computers
}

\author{
Shahla A. Abdul Qadir \\ Technical Institute, Mosul, Iraq
}

Received on: 02/02/2012

\section{ABSTRACT}

Accepted on: 28/06/2012

Efficient technologies have been recently used in Fractal Image Coding (FIC) to reduce the complexity of searching for matching between Range block and Domain block. The research aims at using the Parallel Genetic Algorithm (PGA) by the technology of the (Manager/Worker) in parallel computers to obtain best and quickest compress for images by coding the site of the searching domain block with a Gray code and a fitness function that minimizes the space between the matching of the current range block with the searching domain block in order to choose a protection strategy and compress of high accuracy of images. Results showed that PGA is quicker than standard algorithm in FIC and is more flexible and efficient in reaching the optimum solution in higher speed and efficiency through using the Gray code. The searching method used for the parallel algorithm for compression and decompression, the method of choosing GA's coefficients, (selection, crossover and mutation) were of a significant role in improving the image compression ratio and quality for images in high speed that has reached $15_{\mathrm{s}}$, compression ratio has reached $91.68 \%$, while the image quality was improved after decompression and has reached roughly 34.81 compared to traditional method of fractal image coding (FIC) where the compression ratio has reached $83.87 \%$ and image quality 31.79 with algorithm implementation speed reached $28_{\mathrm{s}}$.

Keywords-Parallel Genetic Algorithm (PGA), Fractal Image Coding(FIC), Local Iterated Function System(LIFS), Rang Block, Domain Block.

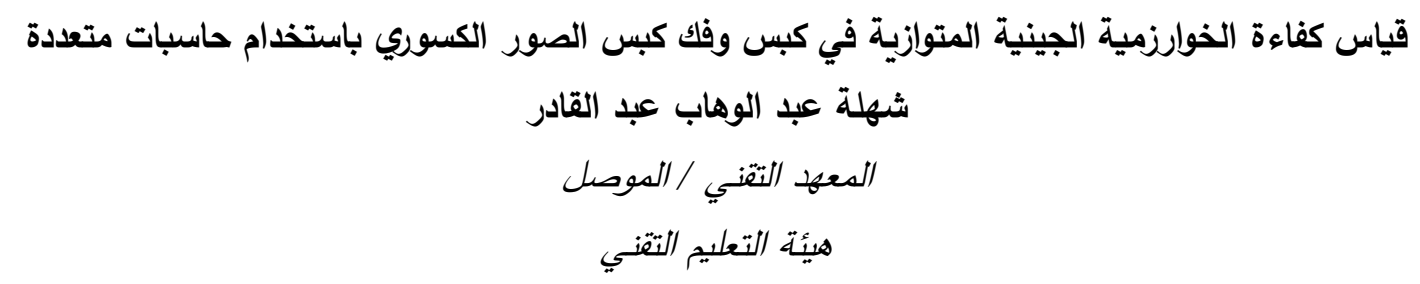

\section{الملخص}

استخدمت مؤخرا تقنيات فعالة في التشفير الكسوري للصور Fractal Image Coding(FIC) لتقليل

تعقيد البحث للتطابق بين كتل المدى Range Block وكتل المجال Domain Block ـ الهدف من هذا البحث

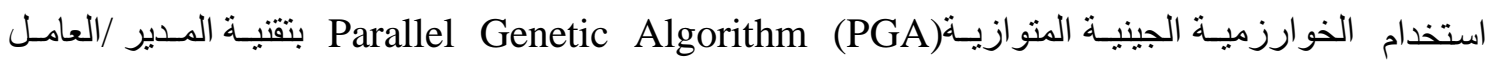
في حاسبات متوازية للحصول على أسرع وأفضل كبس للصور من خلال البحث عن كتل (Manager/Worker) المجال المتطابقة التي تمنع التقارب غير الصحيح بواسطة تثفير موقع كتلة مجال البحث بثفرة رمادية وبدالة لياقة

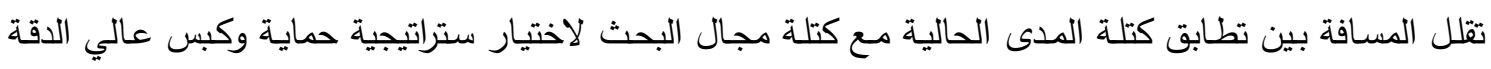

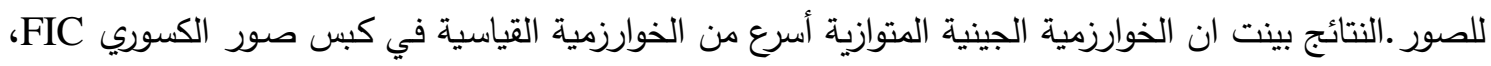

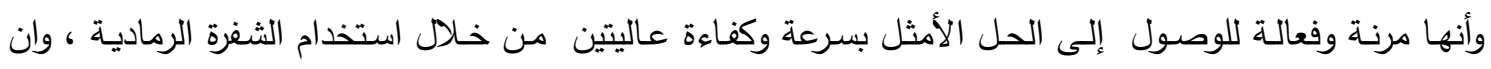

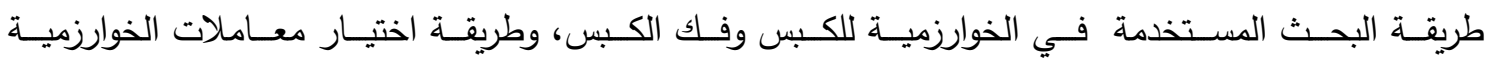


الجينية(الاختيار ،العبور والطفرة) كان له دور فعال في تحسين نسبة الكبس ونوعية الكبس للصورة بسرعة عالية

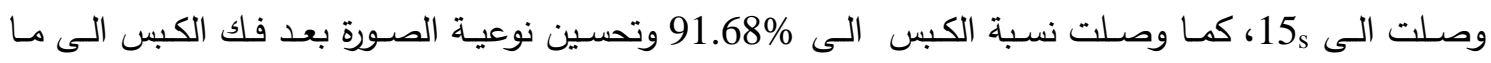

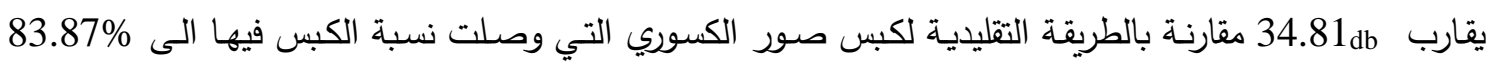

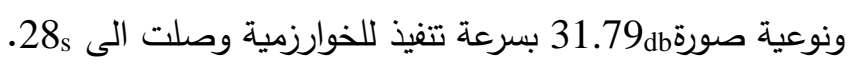
الكلمات المفتاحية: الخوارزمية الجينية المتوازية ، كبس او تثفير الصور الكسوري، نظام الوظيفة المتكرر ، كتلة

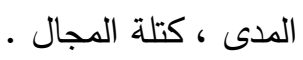

1. المقدمة

كبس وفلك الكبس للصور من تقنيات الصور الرقمية التي أصبحت جانبا مهما في خزنها ونقلها في مجال

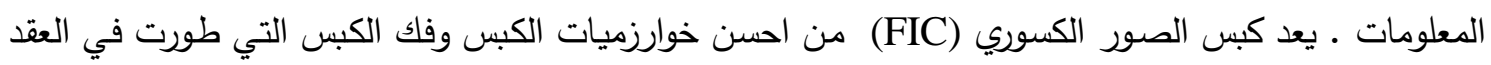

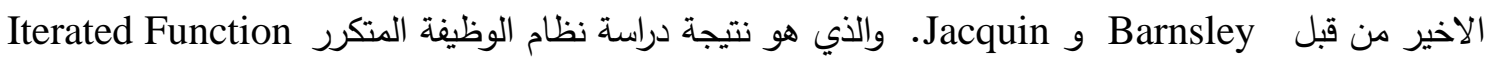

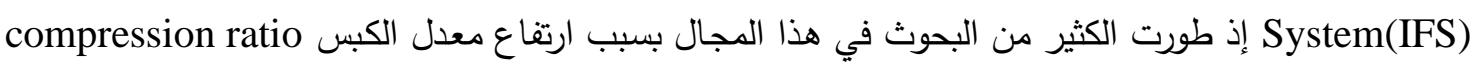

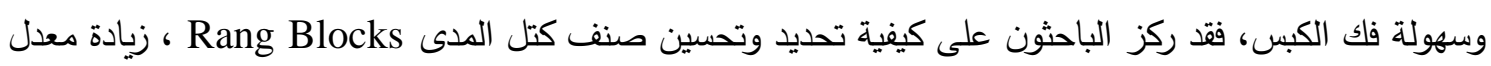

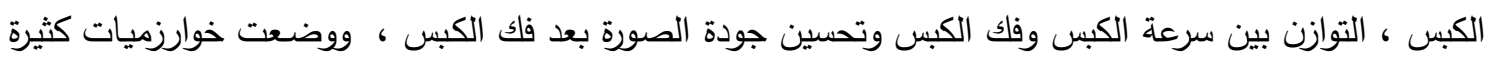

في هذا الدجال على أسـاس تصنيف البحث الذي يقلل التعقيد [1]، فقد استخدم باحثون الخوارزميات الجينة Genetic Algorithm (GA)

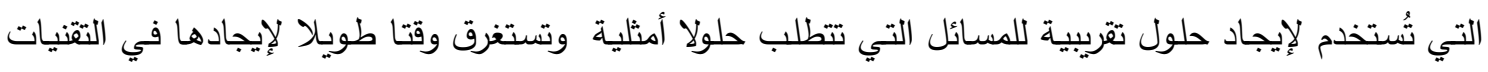
التقليدية ، ويتم في الخوارزمية الجينية توصيف المشكلة بكروموسومات تمثل الحلول بواحدة من طرائق الترميز

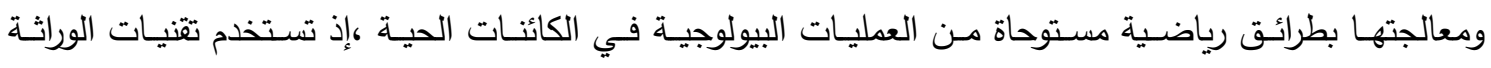

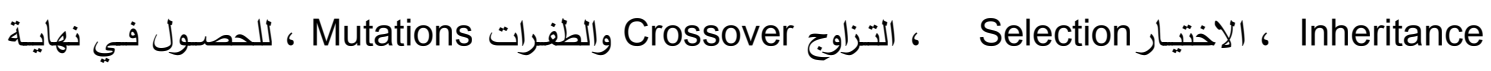
المطاف على الجيل النهائي للكروموسومات والذي يمثل الحل الأمثل للمشكلة المطروحة ، فقد عمل الباحثون [2] [4] [5] [8] [9لى تطوير خوارزميـات جينيـة لكبس الصـور الكسوري وكانت نسبة الكبس عاليـة للصسور

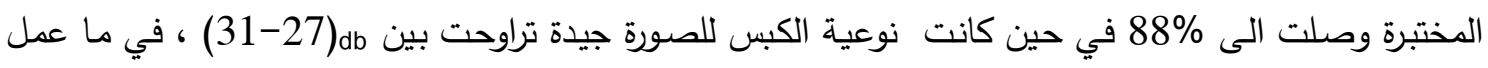
آخرون[3][13][13][13] [13ى تطوير نظم هجينة من الثبكات العصبية والمنطق المضبب والخوارزميات الجينية لكبس الصور الكسوري حيث كانت النتائج متفاوتة بين طريقة واخرى فقد وصلت نسبة الكبس الى

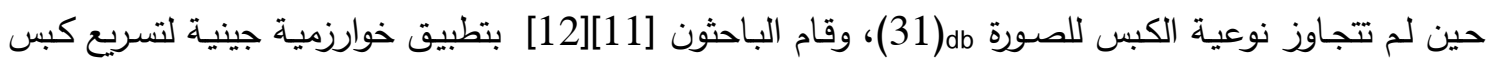

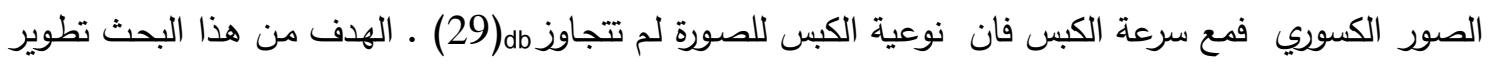
خوارزمية جينية متوازية في حاسبات متوازية لتقليل التعقيد والسرعة من اجل الحصول على كتل المجال المتطابق

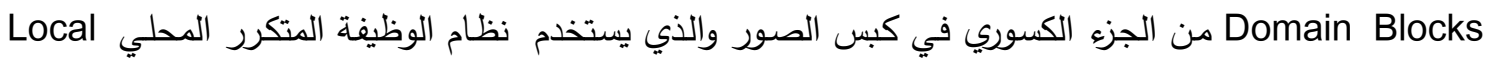
Iterated Function System(LIFS) خوارزمية جينية متوازية بترميز موقع البحث لكتلة المجال وبشفرة رمادية Gray code ذات دالة لياقة لايجاد اقل

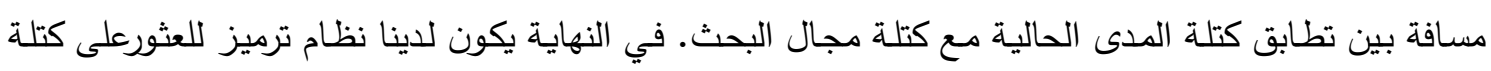
المجال المناسب لكل كتلـة مدى وتوزع على عدة حاسبات لتقليل الوقت المستغرق في حساب دالة اللياقة في 
الخوارزمية الجينية.ماتبقى من البحث كما يلي :القسم الثاني يتضمن تفاصيل الطريقة المستخدمة في البحث. القسم

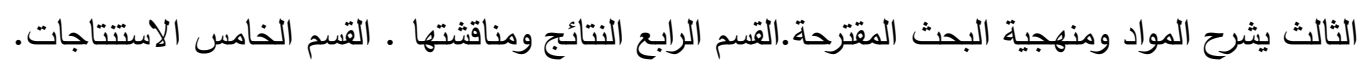

\section{2. الخوارزمية الجينية المتوازية لكبس وفاك الكبس الكسوري}

ستراتيجية الخوارزمية الجينية المتوازية في كبس الصور الكسوري تكون كالاتي:-

2-2 كبس الصور الكسوري (FIC):

كبس الصورة الكسوري عملية معقدة جدا لكن فلك الكبس عملية سهلة جدا مما يجعل معدل الكبس عاليا" فيها ،هذا التعقيد جاء لانها تستند بالاساس على نظرية الوظيفة المتكرر :LISF. ولكبس صورة أصلية بطريقة

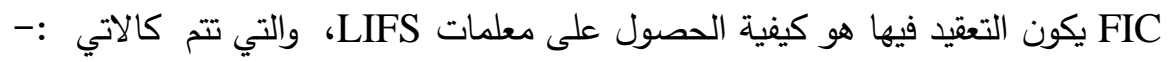

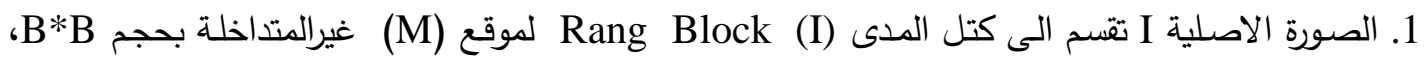
وبشكل عشـوائي لموقع (N) لكتل المجـال Domain Blocks(I) وبحجم 2B*2B. كتـل المـدى ترقم

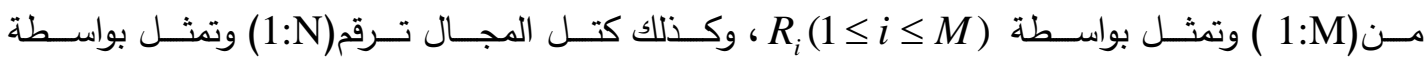

$$
\text { . } D_{j}(1 \leq j \leq N)
$$

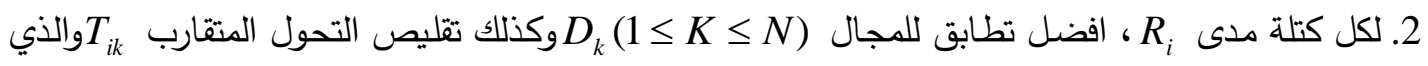

$d\left(R_{i}, T_{i k}\left(D_{k}\right)\right)=\min . d\left(R_{i}, T_{i j}\left(D_{j}\right)\right)$

يحقق بالمعادلة التالية :

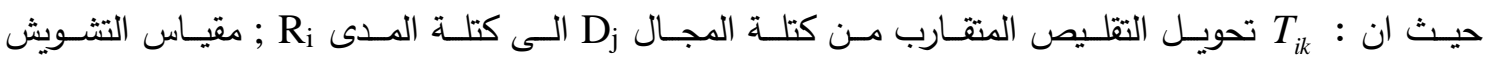

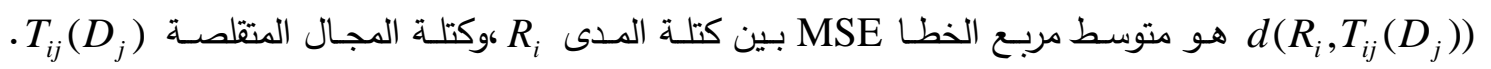
$T_{i j}=\theta_{i j} \circ \phi_{j}$

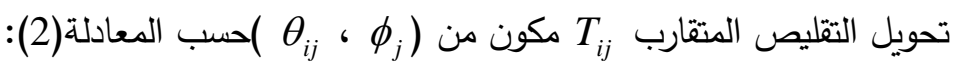

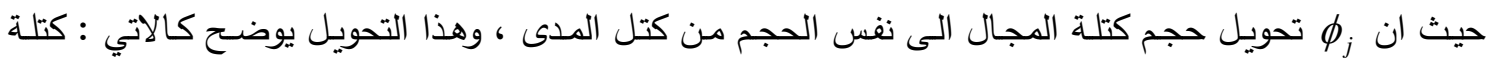

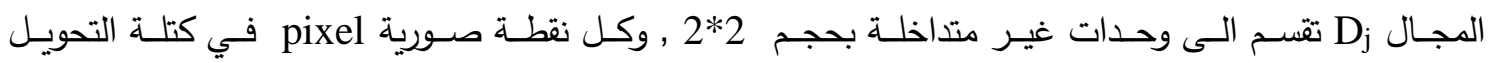

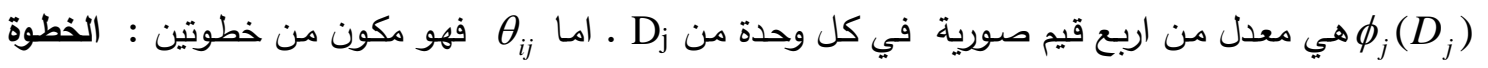
الاولى تحولات كتلة (D)

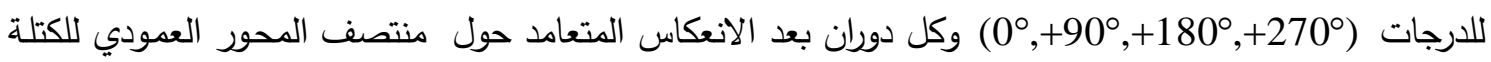

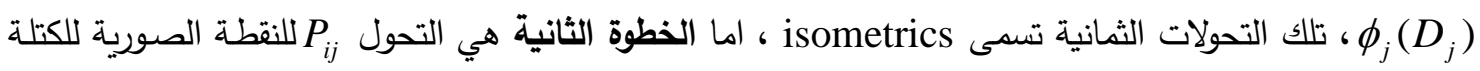

المحصل في الخطوة الاولى ، ويعرف بالمعادلة (3) : $P_{i j}(v)=a_{i j} v+g_{i j}$

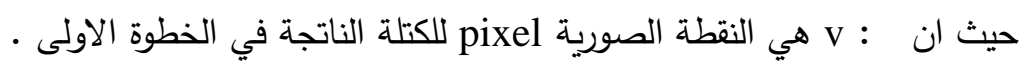

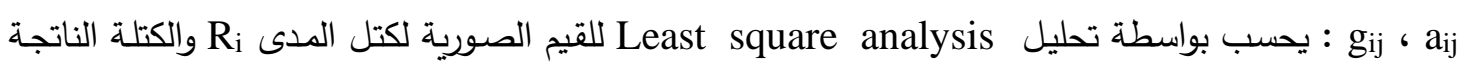

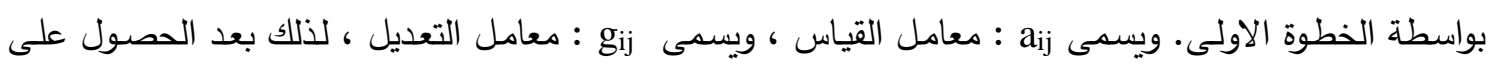
معلمات LIFS يتم تثفيرها كالاتي: 1- معلمات تثير الى موقع افضل تطابق لكتلة المجال. 
2- معلمة تثير الى isometry لـ افضل تطابق لكتلة المجال .

3- معامل القياس

المشكلة الأساسية في هذه الطريقة هو استهلاك وقت كبير في كبس الصور الكسوري، في هذا البحث طورت

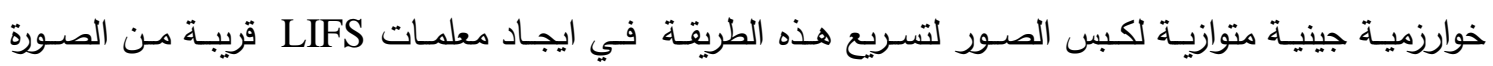

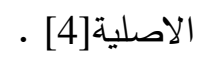

FIC GA 2-2 الخوارزميات الجينية لكبس الصور الكسوري

السمة الرئيسية لـFIC هو ايجاد كتلة المجال المناسبة والتحول الى نوعية من كتلة المدى ، والمشكلة فيها

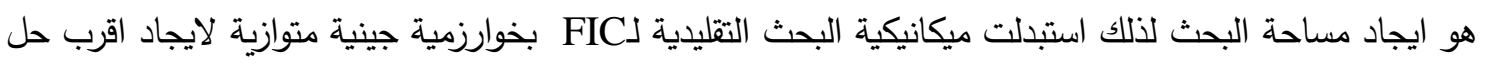

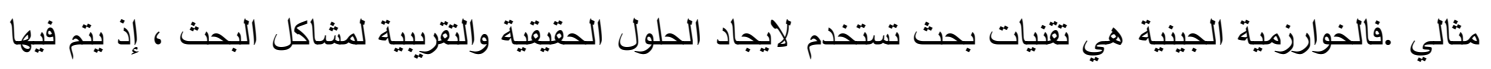

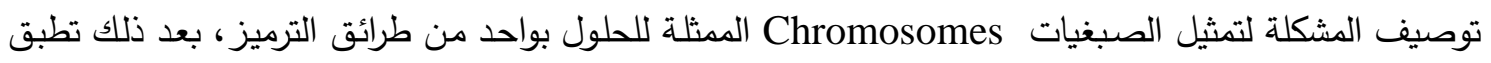

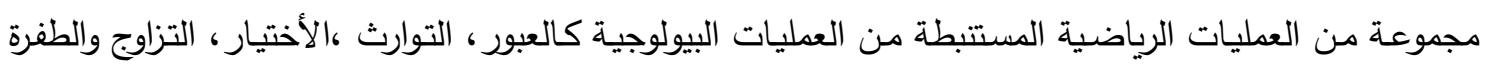

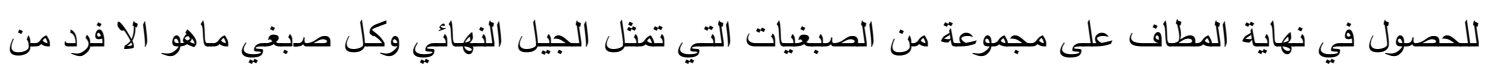

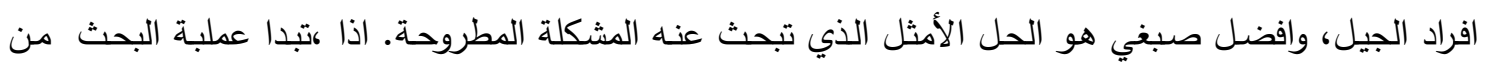
مجموعة حلول وليس حلا'واحدا". الحلول عادة تمثل بصيغة ثنائية (1,0) لكل الاحتمالات الممكنة للمسألة،والجيل الاول يبدا بتوليد جيل عشوائي لكل الصبغيات لتمثيل مساحة الحل. الجيل الجديد يتكون من تقييم كل فرد في

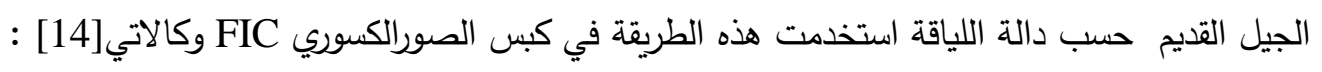

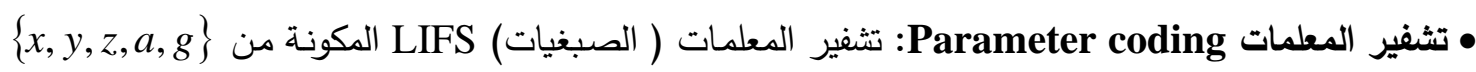

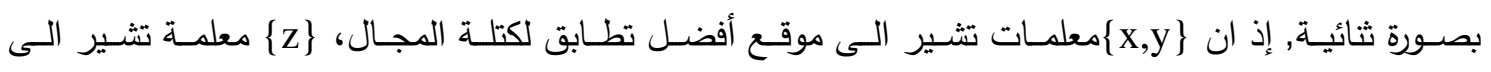

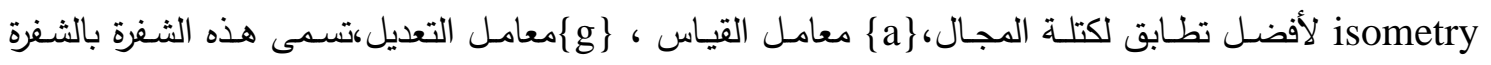
الرمادية للبحث عن كتلة المجال يوضحها الثكل (1) •

\begin{tabular}{|c|c|c|c|c|c|c|c|c|}
\hline $\mathrm{X}_{\mathrm{n}}$ & $\ldots \ldots \ldots$ & $\mathrm{X}_{1}$ & $\mathrm{Y}_{\mathrm{n}}$ & .......... & $\mathrm{Y}_{1}$ & 2 & 1 & 0 \\
\hline
\end{tabular}
الشكل رقم (1): الثفرة الرمادية للبحث عن كتلة المجال

الصورة I(w,w) في عملية الكبس تقسم الى كتل المدى غير المتداخلة بحجم b*b لكل كتلة مدى والبحث

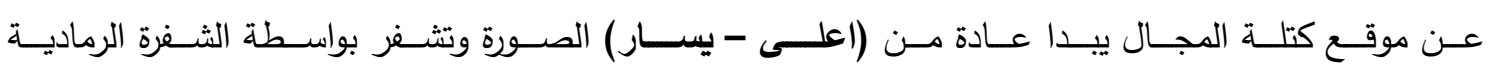

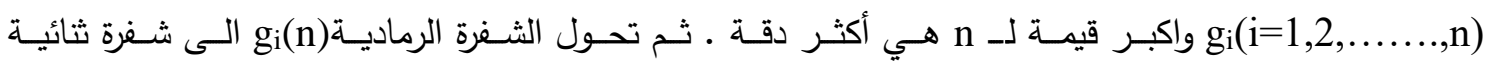
كما في المعادلة(4) X $X_{i}(i=1,2, \ldots, n)$

$X_{n}=g_{n}$ $X_{i}=X_{i+1} \oplus g_{i},(i=n-1, n-2, \ldots \ldots . ., 1)$

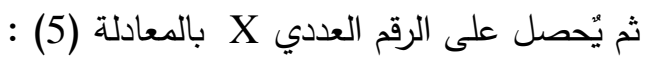

$X=1+\frac{\sum_{i=1}^{n} x_{i} \bullet 2^{i-1}}{2^{n}-1} *(w-2 b)$

• حجم الجيل Population size : يقصد به عدد الأفراد ضمن الجيل وهومن العوامل المهمة والتي يتوقف عليها

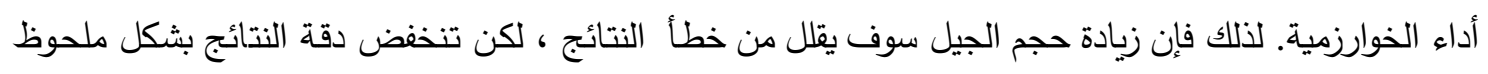


عند كون حجم الجيل صغيرًا لأنه لن يشمل كامل المجال المدروس بشكل جيد ،وتتم عملية بناء الجيل الابتدائي initial population

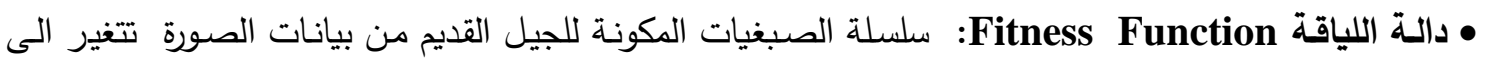
الجيل الجديد اعتمادا على دالة اللياقة بالمعادلة (6) :

$\operatorname{Fitness}(x)=\left\{\begin{array}{l}\frac{1}{m s e(x)+1} \ldots m s e(x)>\varepsilon \\ \infty \ldots \ldots \ldots \ldots \ldots \ldots . . m s e(x) \leq \varepsilon\end{array}\right\}$

حيث MSE(x) هي متوسط مربع الخطأ بين كتلة المدى وكتلة المجال الناتجة من الصبغي X • توليد الاجيال ولت Generation: توليد الأجيال يعني الحصول على جيل جديد من الابناء من خـلال توالد صبغيات الاباء التي لها لياقة اكبر وفرصة للتزاوج والبقاء، وبهذا يتم توليد جيل جديد من الابناء بثكل عشوائي اكثر تكيفا للظروف البيئية وفناء الافراد الاقل تكيفا ،لذلك كلما زاد عدد الاجيال فان الحلول المثلى سوف تحافظ

على نفسها في الاجيال اللاحقة باقل تقدير ، وتمر عملية التوليد بثلاث مراحل، ( الاختيار ، التزاوج ، الطفرة).

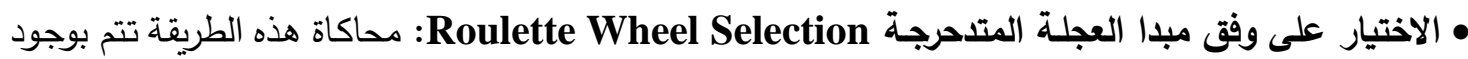

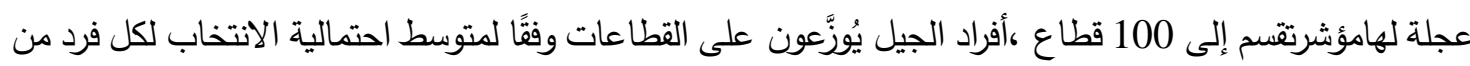
أفراد الجيل الحالي والذي يعطى بالمعادلة (7) : Pis $=F i /\left(\sum_{i=1}^{m} F i\right),(i=1,2, \ldots \ldots \ldots . M)$

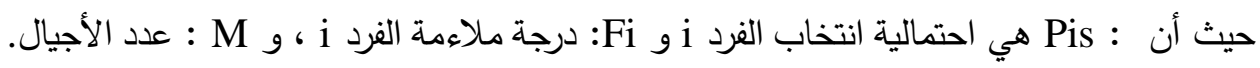

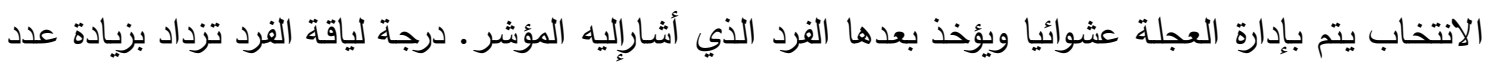
قطاعاته ومن ثم يزاد أحتمال انتخابه في الجيل اللاحق بتأثير إيجابي.

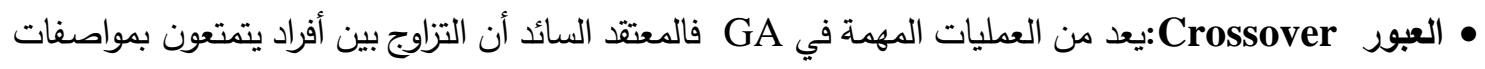

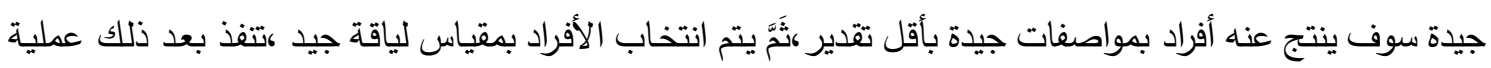

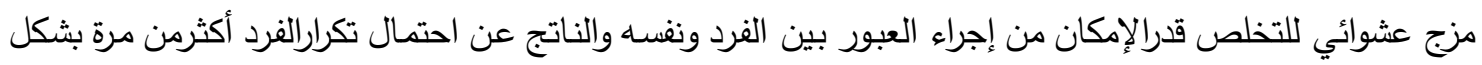
متتالٍ نتيجة عملية الانتخاب التي تسبق العبور .في جيل الاباء تقسم الصبغيات الى( ثلاث مجاميع) والعبوريتم فيها

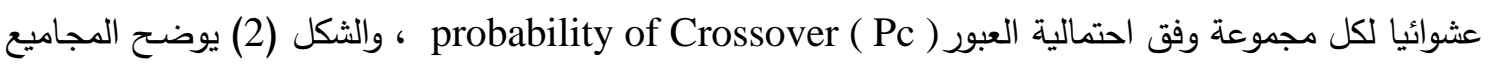

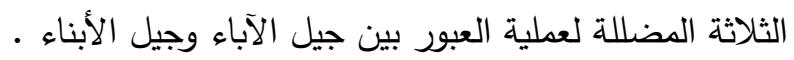
• الطفرة Mutation : هي المرحلة الأخيرة من سلسلة العمليات التكرارية التي تسهم بشكل جيد للوصول إلى الحل

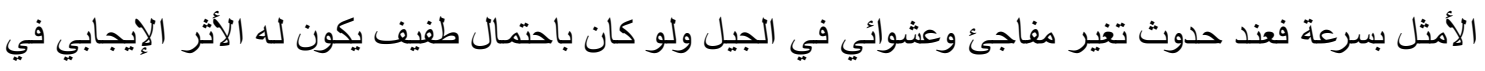

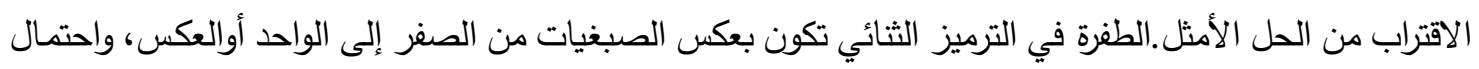
حدوث هذا التغير يعرف باحتمال الطفرة ( Pm و ون عادة يختار بقيمة متدنية

نسبيًا [14]

\begin{tabular}{|c|c|c|c|c|c|c|c|c|c|c|c|c|c|c|c|c|c|}
\hline \multirow{2}{*}{ الآجاءو } & $\mathrm{X}_{\mathrm{l}, \mathrm{n}}$ & $\mathrm{X}_{1, \mathrm{n}-1}$ & ... & $\mathrm{X}_{\mathrm{1}, \mathrm{j}}$ & $\mathbf{X}_{1, j-1}$ & & $\mathbf{X}_{1,1}$ & $\mathbf{Y}_{1, \mathrm{n}}$ & $Y_{1, n-1}$ & .. & $\mathbf{Y}_{1, \mathrm{j}}$ & $\mathbf{Y}_{1, j-1}$ & & $\mathbf{Y}_{1,1}$ & $\mathrm{Z}_{1,1}$ & $\mathrm{Z}_{12}$ & $\mathrm{Z}_{1,3}$ \\
\hline & $\mathrm{X}_{2, \mathrm{n}}$ & $\mathrm{X}_{2, \mathrm{n} \cdot \mathrm{l}}$ & . & $\mathrm{X}_{2, \mathrm{j}}$ & $\mathbf{X}_{2, j-1}$ & " & $\mathbf{X}_{2,1}$ & $Y_{2, n}$ & $Y_{2, n-1}$ & $"$ & $Y_{2, j}$ & $\mathbf{Y}_{2, j-1}$ & ". & $\mathbf{Y}_{2,1}$ & $\mathbf{Z}_{2,1}$ & $\mathbf{Z}_{22}$ & $\mathbf{Z}_{2,3}$ \\
\hline \multirow{2}{*}{ الأبناء } & $\mathrm{X}_{1, \mathrm{n}}$ & $\mathrm{X}_{1, \mathrm{n}-1-1}$ & $\ldots$ & $\mathrm{X}_{\mathrm{1}, \mathrm{j}}$ & $\mathbf{X}_{2, j-1}$ & .. & $\mathrm{X}_{2,1}$ & $\mathbf{Y}_{1, \mathrm{n}}$ & $Y_{1, n-1}$ & & $\mathbf{Y}_{2, \mathrm{j}}$ & $\mathbf{Y}_{2, j-1}$ & & $\mathbf{Y}_{2,1}$ & $\mathrm{Z}_{1,1}$ & $\mathrm{Z}_{12}$ & $\mathbf{Z}_{2,3}$ \\
\hline & $\mathrm{X}_{2, \mathrm{n}}$ & $\mathrm{X}_{2, \mathrm{n}-1}$ & $\ldots$ & $\mathrm{X}_{2, \mathrm{j}}$ & $\mathbf{X}_{1, j-1}$ & . & $\mathrm{X}_{1,1}$ & $Y_{2, \mathrm{n}}$ & $Y_{2, n-1}$ &. & $Y_{1, j}$ & $\mathbf{Y}_{1, j-1}$ & 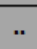 & $\mathbf{Y}_{1,1}$ & $\mathbf{Z}_{2,1}$ & $\mathbf{Z}_{22}$ & $\mathrm{Z}_{1,3}$ \\
\hline
\end{tabular}

الشكل رقم (2): العبور للشفرة الرمادية 


\section{3-2 3 الخوارزمية الجينية المتوازية}

الخوارزمية الجينية تتطلب وقتا'طويلا'لتنفيذها لكن في تقنيات الحاسوب الحديث تم التغلب على طول الوقت

بتتفيذ هذا النوع مـن الخوارزميات في حاسبات متوازيـة الهدف منـه الحصول على الحل الأمثل بسرعة عالية. الخوارزمية الجينية المتوازية طبقت على أساس المدير / العامل Manager/Worker/بعدة حاسبات،حاسبة رئيسية تعد المدير ،و N من الحاسبات الطرفية بوصفها عمالا "،الموضحة بالثكل (3). المدير يعمل على تأسيس الاتصال مع العمال من خلال إرسال إثارة الاتصال،ثم يقوم بتوليد صبغيات الجيل الابتدائي عشوائيا وتوزيعها على العمال بالتسـاوي ، تهيئة متطلبـات الخوارزميـة الجينيـة وتوزيعها على العمال Worker-N.أيضـا يقوم المدير باستلام

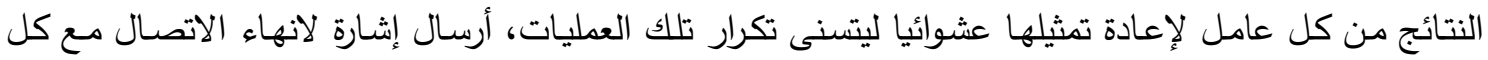
العمال بعد الحصول على الحل الأمثل النهائي. أما العمال N ،يقوم كل عامل من البداية باستلام الإشارة من المدير لتأسيس الاتصال والانتظار لحين البدء بإرسال متطلبات الخوارزمية الجينية ،وجزء من الصبغيات الكلية لتتفيذ الخوارزمية بدالة اللياقة ، ومن ثم إرسال ناتج الخوارزمية الجزئية الى المدير،العمليات تكرر لكل عامل مـع كل إرسال من المديرإلى أن يستلم إثارة بإنهاء الاتصـال بعد الوصول إلى الحل الأمثل النهائي [6][7].

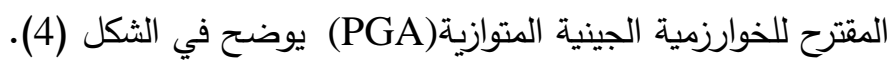

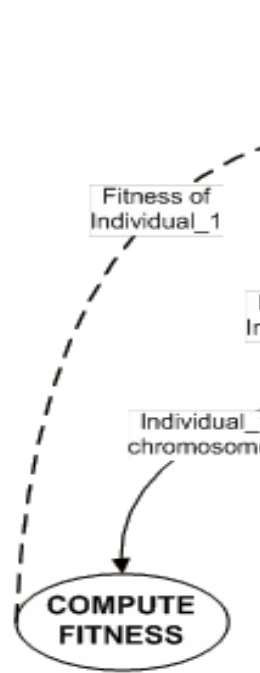

WORKER 0
MANAGER SELECTION MUTATION NEW POPULATION

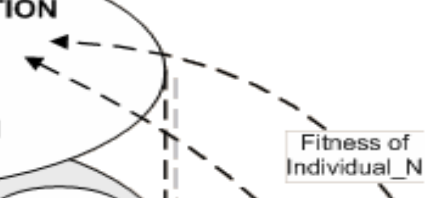

Fitness of Individual__

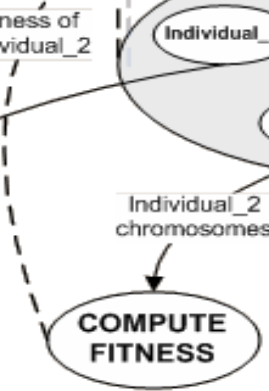

WORKER 1

الشكل (3) الخوارزمية الجينية المتوازية 


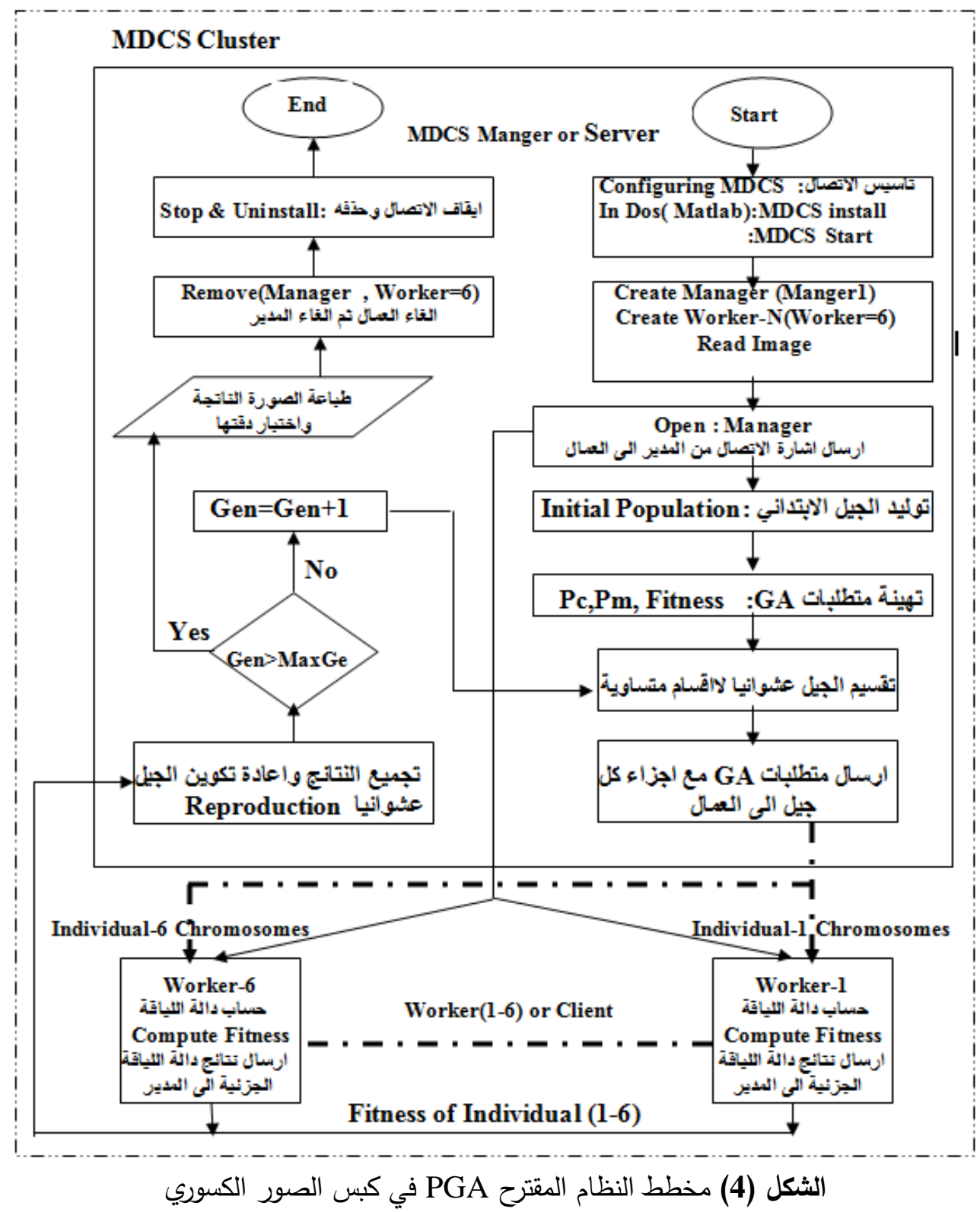

3. 3واد البحث وطرائقه :

وفقا لما تقدم في المقطع الثاني ، استخدمت بيانات الصور الأصلية الرمادية (Lena ,Barbara)

بصيغة (JPEG) لكونها صيغة فعالة لضغط الصور الرقمية وهي الصيغة الاكثر شيوعا لحفظ وارسال ملفات الصور عبر شبكة الانترنت لان حجمها صغير ودقتها عالية ـ واختيرت الصور بحج256*256 والتي شفرت ب 8-bit/pixel لكل نقطة صورية والجدول رقم (1) يمثل البيانات المستخدمة في تمثيل أنموذج الخوارزمية الجينية المتوازية ـ استخدمت تقنية MATLAB Distributed Computing Server (MDCS) المعروفة بخوادم الحاسبات الموزعة والموجودة في بيئة الماتلاب MatlabR2010b وتأسيسها على مجموعة حاسبات ـ واستخدم منها تقنية المدير/العامل Manager/Worker ، حيث ربطت (7) حاسبات ،واحدة منها تمثل المدير يعمل على لمعلى

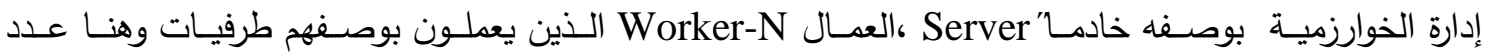
الحاسبات :Worker-N=6،حيث تم تاسيس الاتصال كالاتي : 
1-Install the MDCE Service on all computers

2-In Dos for the folder:

Matlabrootldistcomplbin\Mdce install

Matlabrootldistcomplbin $\backslash$ Mdce start

Matlabrootldistcomplbin $\backslash$ admincenter.bat

3-In admincenter: Create Job Manager : Jm1, Worker (1 - 6)

Imnosie(image Name ,'salt \& pepper',d)

كما تم اضافة اخطاء للصور باستخدام الايعاز الآتي وبنسب مختلفة : حيث ان :image Name : اسم الصورة المضاف اليها noise. on and off 'salt \& pepper' noise density وهي : D

واستخدمت المعادلات (8)،(9) لقياس كفاءة الخوارزمية المقترحة من خلال الايعاز الآتي: [PSNR,MSE,Error, Compression raio ] = measerr(image Name)

$$
\begin{gathered}
M S E=\frac{\sum_{i=0}^{M_{\text {Rows }}} \sum_{j=0}^{N_{\text {Cols }}}\left|X(i, j)-X_{c}(i, j)\right|^{2}}{M_{\text {Rows }} * N_{\text {Cols }}} \\
P S N R=10 \log _{10}\left(\frac{255^{2}}{M S E}\right)
\end{gathered}
$$

\begin{tabular}{|c|c|}
\hline 50 & 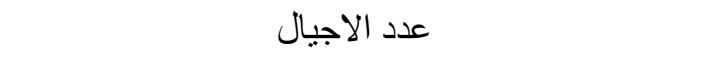 \\
\hline 240,150 & حجم افر اد الجيل الكلي المولد في المدير Manager \\
\hline 40,25 & حجم الجيل المستلم لكل عامل Worker \\
\hline معادلة(6) & 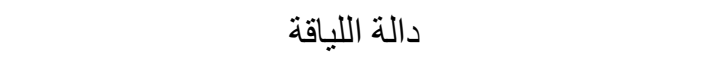 \\
\hline 1 & احتمالية العبور المثالية (Pc) \\
\hline 0.001 & احتمالية الطفرة المثالية (Pm) \\
\hline $4 * 4$ & حجم كتلة المدى \\
\hline
\end{tabular}

الجدول رقم (1) بيانات أنموذج الخوارزمية الجينية المتوازية

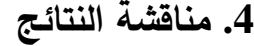

تشير النتائج التي حصل عليها أن تطبيق الخوارزمية الجينية المتوازية لها تأثير في تحسين عملية الكبس وفك الكبس الكسوري FIC وبسرعة أفضل من الطريقة القياسية لكبس الصور • تبين ذلك من خلال استخدام صور

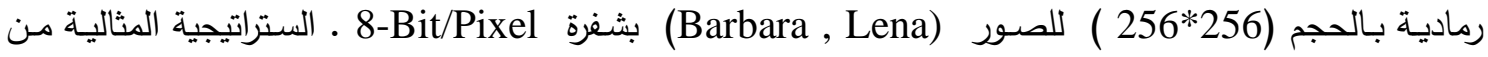
النقط الصورية ( Pixel ) للشفرة الرمادية لفضـاء البحث مكونـة من 14-bit لكتلة المجال المتطابق (باحداثيات عمودية وافقية ) ، 3-bit للمعلمة 5-bit ، isomorphic لمعلمة القياس ، 7-bit لمعلمة التعديل • ولكل كتلة مدى الثفرة الكسورية مكونة من bit-29 وتخزن في ملف نصي يسمى ملف الكبس. من ناحية اخرى خلال عملية التكرار لفك الكبس تم التغلب على قيم الصورة الرمادية اذا تجاوزت المدى (0-256) باخذ معدل النقاط الصورية الأربعة الدجاورة لتجنب التباعد عن القيم الأصلية ـ النتائج قيست على أساس سرعة تتفيذ الخوارزمية الجينية في 
حاسبات متعددة فعند اختيارحجم الجيل 240 وعدد مرات التكرار 50 مرة ، وبتنفيذات مختلفة يوضحها الجدول (2)

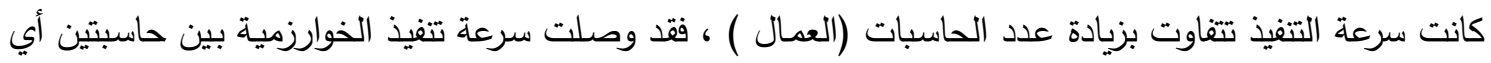

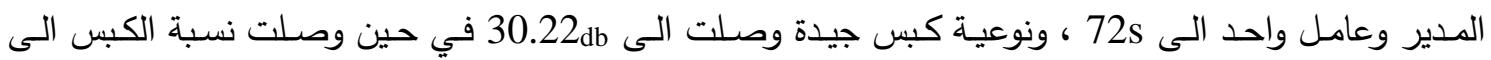

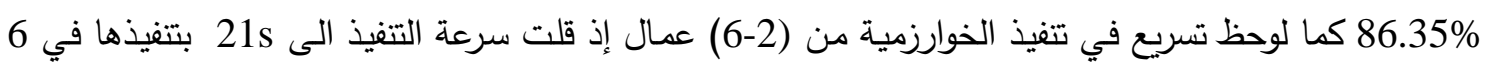

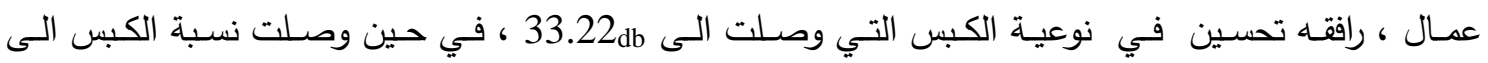

الجدول رقم (2): نتائج صورة Lena بحجم جيل (150, 240) ، (6) عمال

\begin{tabular}{|c|c|c|c|c|c|}
\hline نompression ratio \% & الكبسية & $\begin{array}{c}\text { الموفُتْ } \\
\text { Time(S) }\end{array}$ & Iteration & $\begin{array}{c}\text { حجم الجيل } \\
\text { Population size }\end{array}$ & عداد العمال \\
\hline 86.35 & 30.22 & 72 & 50 & 240 & 1 \\
\hline 86.71 & 30.87 & 66 & 50 & $=$ & 2 \\
\hline 87.21 & 31.50 & 51 & 50 & $=$ & 3 \\
\hline 87.44 & 32.33 & 39 & 50 & $=$ & 4 \\
\hline 88.77 & 33.17 & 28 & 50 & $=$ & 5 \\
\hline 89.22 & 33.22 & 21 & 50 & $=$ & 6 \\
\hline 88.16 & 32.88 & 68 & 50 & 150 & 1 \\
\hline 88.65 & 32.67 & 50 & 50 & $=$ & 2 \\
\hline 89.36 & 33.12 & 41 & 50 & $=$ & 3 \\
\hline 89.65 & 33.45 & 31 & 50 & $=$ & 4 \\
\hline 89.87 & 33.87 & 22 & 50 & $=$ & 5 \\
\hline 90.12 & 34.21 & 16 & 50 & $=$ & 6 \\
\hline
\end{tabular}

كذلك لوحظ ان هناك تسريعا'في تتفيذ الخوارزمية الجينية المتوازية عند تقليل حجم الجيل ، فعند تقليل حجم الجيل من 240 الى 150 تراوح وقت التتفيذ بين s(68-16) لكل حالات التتفيذ الموضحة بالجدول

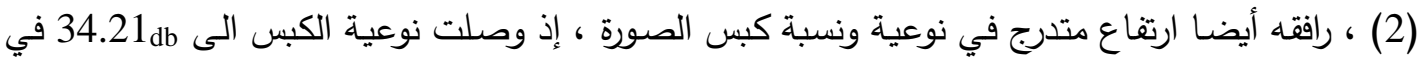
حين وصلت نسبة الكبس الى190.12\% باسرع تنفيذ للخوارزمية بعدد عمال مساوٍ لـ 6. ولغرض اختبار دقة النتائج التي طبقت على صورة Lena تم تطبيق الخوارزمية على صورة Barbara وبنفس المحددات ، فقد لوحظ ان هنالك تسريعا"في كبس وفك الكبس باستخدام PGA يوضحها الجدول (3)، فعند اختيار حجم الجيل 240 وبتكرار 50مرة كانت سرعة التنفيذ تتفاوت بزيادة عدد الحاسبات ( العمال) ، إذ وصلت سرعة التفيذ الى بلى 6 22s بعدد العمال 6 رافقه ايضا تحسين في نوعية الكبس للصورة التي وصلت الى 33.20db في حين وصلت نسبة الكبس الى 89.17\% .كذلك لوحظ ان هنالك تسريعا'في تتفيذ الخوارزمية عند تقليل حجم الجيل الى 150 إذ وصلت سرعة التتفيذ الى 17 بنوعية كبس للصورة وصلت الى 34.12db ونسبة كبس مساوٍ الى لى 
الجدول رقم (3) : نتائج صورة Barbara بحجم جيل(240 , 150 ), ( 6 ) عمال

\begin{tabular}{|c|c|c|c|c|c|}
\hline $\begin{array}{c}\text { نسبة الكبس } \\
\text { Compression } \\
\text { ratio\% }\end{array}$ & نويسة & $\begin{array}{c}\text { الّوقت } \\
\text { Time(S) }\end{array}$ & $\begin{array}{c}\text { التكرار } \\
\text { Iteration }\end{array}$ & $\begin{array}{r}\text { حجم الجيل } \\
\text { Population } \\
\text { size }\end{array}$ & عدد العمال \\
\hline 86.31 & 30.17 & 73 & 50 & 240 & 1 \\
\hline 86.69 & 30.83 & 67 & 50 & $=$ & 2 \\
\hline 87.18 & 31.46 & 49 & 50 & $=$ & 3 \\
\hline 87.39 & 32.21 & 40 & 50 & $=$ & 4 \\
\hline 88.70 & 33.11 & 27 & 50 & $=$ & 5 \\
\hline 89.17 & 33.20 & 22 & 50 & $=$ & 6 \\
\hline 88.11 & 32.83 & 69 & 50 & 150 & 1 \\
\hline 88.59 & 32.53 & 52 & 50 & $=$ & 2 \\
\hline 89.31 & 33.11 & 43 & 50 & $=$ & 3 \\
\hline 89.59 & 33.31 & 34 & 50 & $=$ & 4 \\
\hline 89.81 & 33.76 & 21 & 50 & $=$ & 5 \\
\hline 90.01 & 34.12 & 17 & 50 & $=$ & 6 \\
\hline
\end{tabular}

من الجدولين (2) (3) يلاحظ انه مع بقاء التكرار ثابتا'حصل تسريع في تتفيذ الخوارزمية ووصل الى

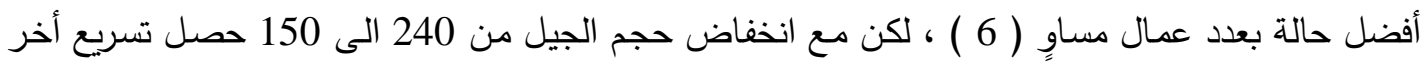

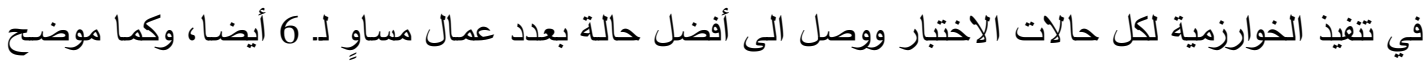

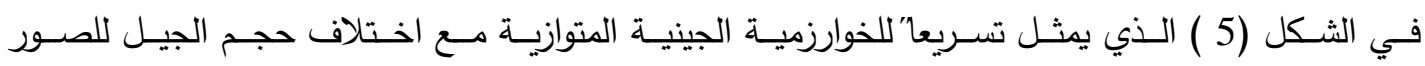

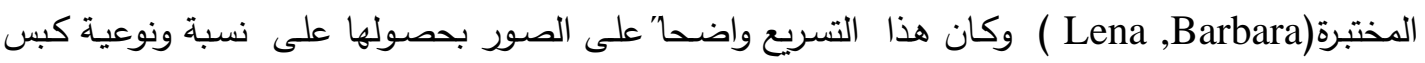

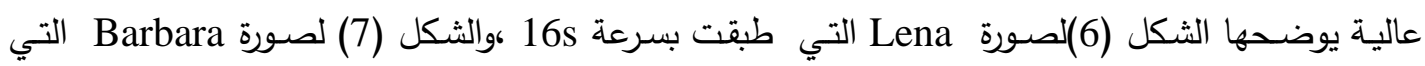

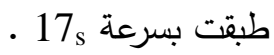

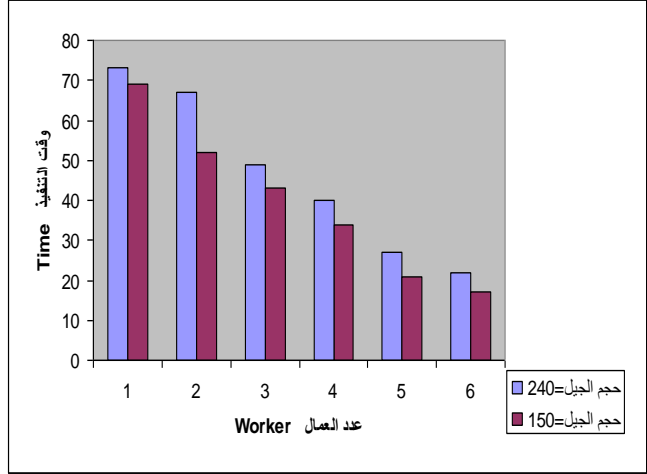

Barbara لصورة PGA تسريع (B)

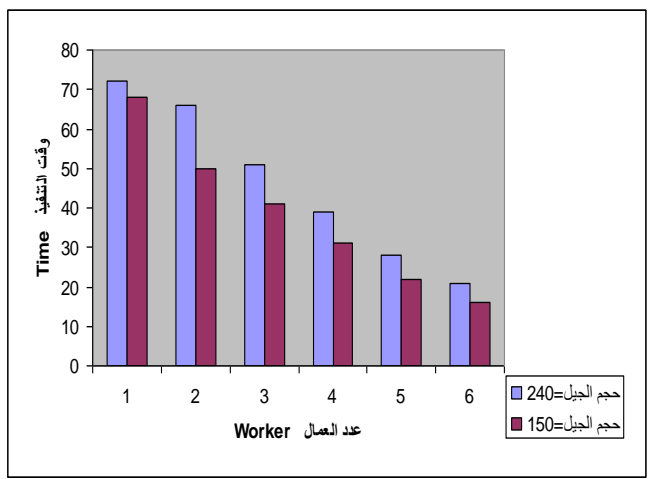

Leana لصور PGA تسريع (A)

الثكل (5) الوقت المستغرق لكبس الصور الكسوري باستخدام PGA في( 6 عمال) وبحجم جيل(240,150) 


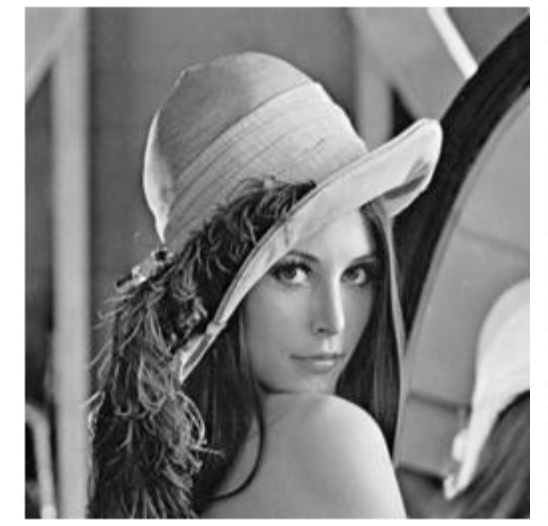

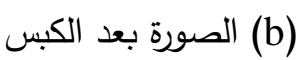

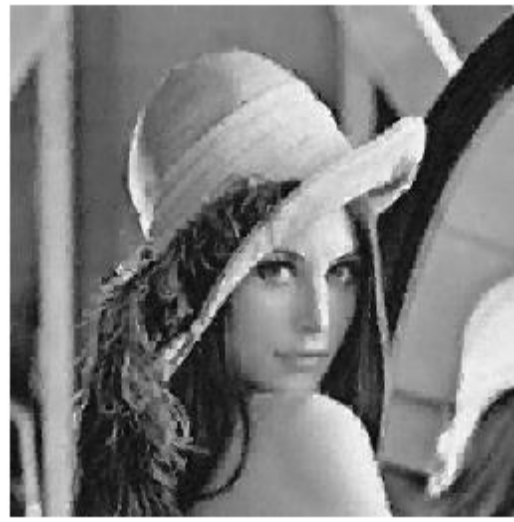

كان (a) (a) (a) (الصورة الاصلية

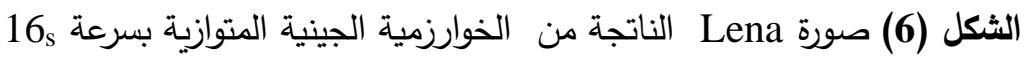

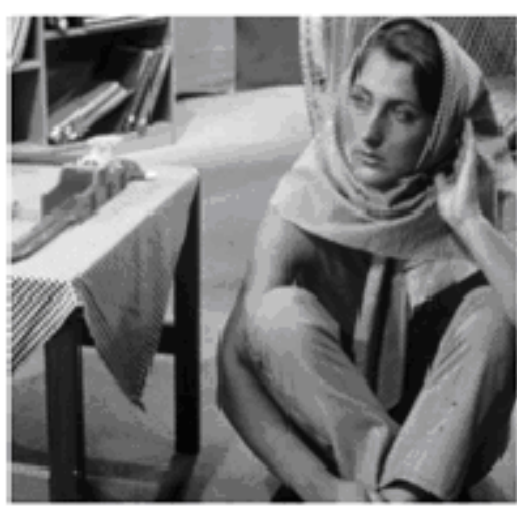

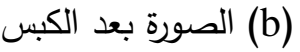

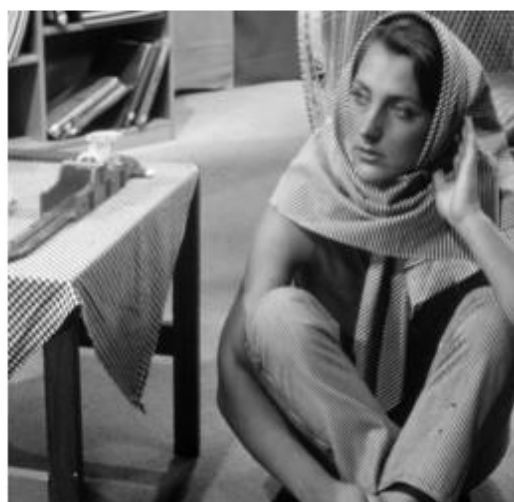

(a) (a) (a) (a) (الصورة الاصلية

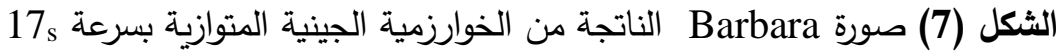

ولغرض قياس كفاءة هذه الطريقة تم مقارنتها مع الطريقة القياسية الكسورية من خلال إضافة أخطاء

للصـور وبنسـب معينـة واختبرت بـالطريقتين والموضـحة بالجدول (4) و(5) إذ تبـين ان الطريقـة القياسـية

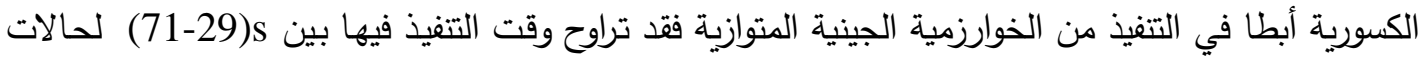

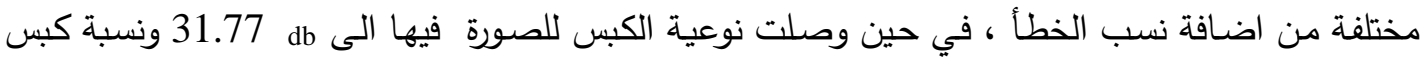
الى 83.85\% لصورة Lena في حين تراوح وقت التنفيذ لصورة Barbara s28 كما وصلت نوعية الكبس الى , 31.79db ونسبة كبس وصلت الى 83.87\% ـ وحصل تسريع للخوارزمية الجينية المتوازية مع

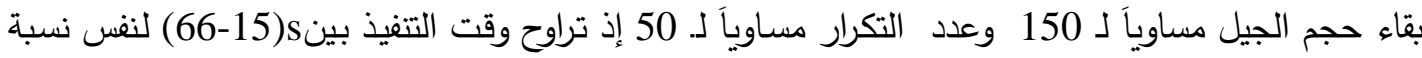
الخطا المضافة لصورةLena في الطريقة القياسية، مع ارتفاع متدرج لنسبة ونوعية الكبس للصورة، فقد كان

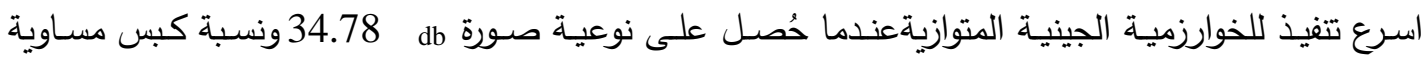

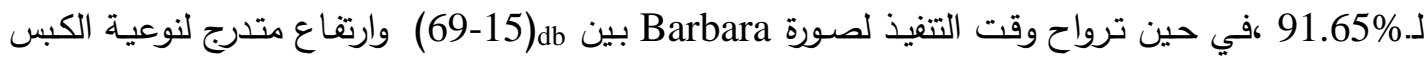
التي وصلت الى db db.81 ونسبة كبس وصلت الى 91.68\% ، وافضل نوعية صورة وأحسنها من ناحية

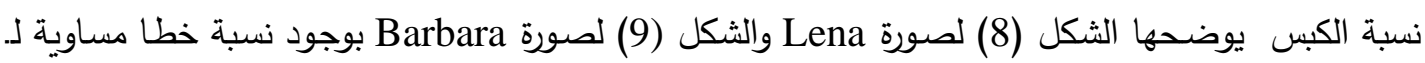

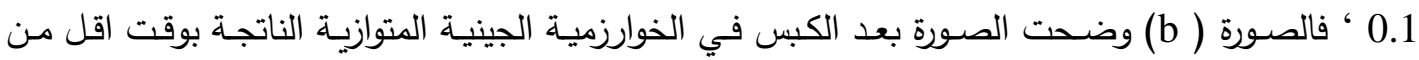

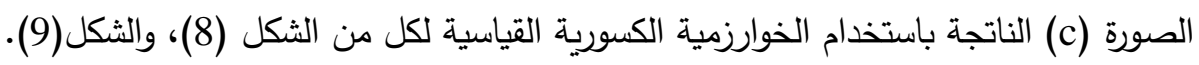


الجدول رقم (4) : نتائج صور (Barbara، Lena ) بعد إضافة نسبة خطا في PGA

\begin{tabular}{|c|c|c|c|c|c|c|c|}
\hline $\begin{array}{c}\text { نسبة الكبس } \\
\text { Compression } \\
\text { ratio\% }\end{array}$ & الكبس & $\begin{array}{c}\text { الوقَت } \\
\text { Time(S) }\end{array}$ & $\begin{array}{c}\text { التكرار } \\
\text { Iteration }\end{array}$ & $\begin{array}{l}\text { نسبة خطا } \\
\text { Error }\end{array}$ & $\begin{array}{c}\text { حجم الجزل } \\
\text { Population size }\end{array}$ & $\begin{array}{l}\text { عدد العمال } \\
\text { workers }\end{array}$ & اسم الصورة \\
\hline 88.77 & 32.89 & 66 & 50 & 0.01 & 150 & 1 & \multirow{6}{*}{ Lena } \\
\hline 88.98 & 32.96 & 49 & 50 & 0.03 & $=$ & 2 & \\
\hline 89.78 & 33.58 & 37 & 50 & 0.06 & $=$ & 3 & \\
\hline 89.98 & 33.88 & 28 & 50 & 0.08 & $=$ & 4 & \\
\hline 90.44 & 34.34 & 20 & 50 & 0.09 & $=$ & 5 & \\
\hline 91.65 & 34.78 & 15 & 50 & 0.1 & $=$ & 6 & \\
\hline 88.79 & 32.91 & 69 & 50 & 0.01 & 150 & 1 & \multirow{6}{*}{ Barbara } \\
\hline 89 & 32.97 & 50 & 50 & 0.03 & $=$ & 2 & \\
\hline 89.80 & 33.61 & 40 & 50 & 0.06 & $=$ & 3 & \\
\hline 89.99 & 33.91 & 29 & 50 & 0.08 & $=$ & 4 & \\
\hline 90.47 & 34.37 & 22 & 50 & 0.09 & $=$ & 5 & \\
\hline 91.68 & 34.81 & 15 & 50 & 0.1 & $=$ & 6 & \\
\hline
\end{tabular}

الجدول رقم (5) : نتائج صور ( Barbara، Lena ) بعد إضافة نسبة خطا في الخوارزمية الكسورية القياسية

\begin{tabular}{|c|c|c|c|c|}
\hline $\begin{array}{l}\text { نسبة الكبنس } \\
\text { Compression } \\
\text { ratio\% }\end{array}$ & نوعية & $\begin{array}{c}\text { الوقت } \\
\text { Time(S) }\end{array}$ & خطبة & الصورة \\
\hline 80.98 & 22.76 & 71 & 0.01 & \multirow{6}{*}{ Lena } \\
\hline 81.89 & 27.44 & 57 & 0.03 & \\
\hline 82.88 & 29.55 & 48 & 0.06 & \\
\hline 83.11 & 30.11 & 38 & 0.08 & \\
\hline 83.55 & 30.56 & 36 & 0.1 & \\
\hline 83.85 & 31.77 & 29 & 0.2 & \\
\hline 80.99 & 22.78 & 72 & 0.01 & \multirow{6}{*}{ Barbara } \\
\hline 81.92 & 27.46 & 59 & 0.03 & \\
\hline 82.92 & 29.57 & 49 & 0.06 & \\
\hline 83.17 & 30.15 & 37 & 0.08 & \\
\hline 83.58 & 30.58 & 37 & 0.1 & \\
\hline 83.87 & 31.79 & 28 & 0.2 & \\
\hline
\end{tabular}




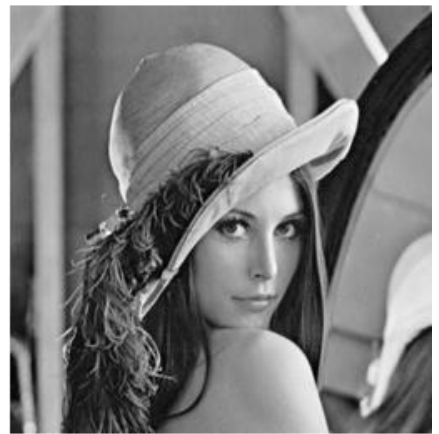

FIC (c)

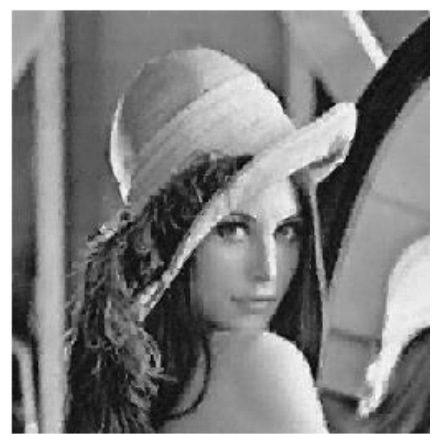

PGA باستخدام FIC (b)

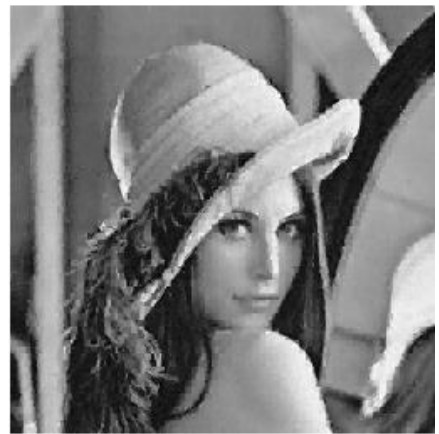

الثكل (a) الصورة الاصلية

الثكل (8) افضل نوعية ونسبة كبس لصورة Lena الناتجة بنسبة خطا (0.1)

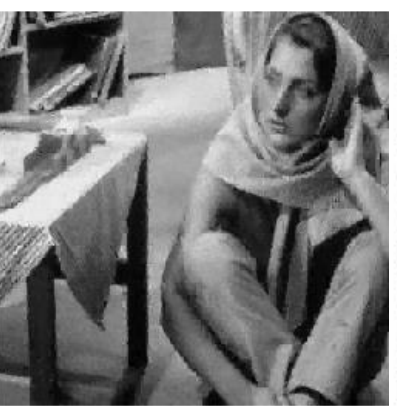

FIC (c)

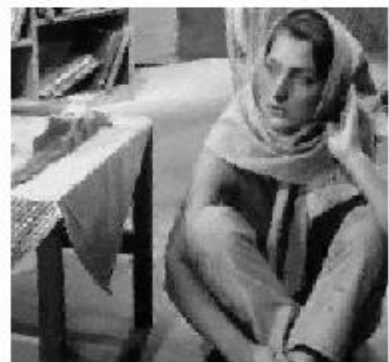

PGA باستخدامFIC (b)

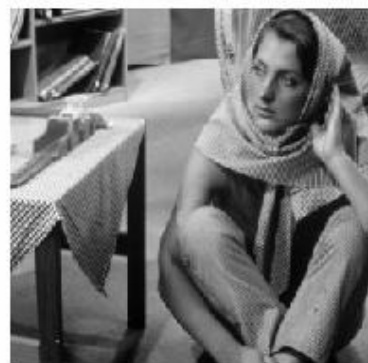

الثكل (a) الصورة الاصلية

الثكل (9) افضل نوعية ونسبة كبس لصورة Barbara الناتجة بنسبة خطا(0.1 )

$$
\text { 5. الاستتتاجات والتوصيات }
$$

الخوارزمية الجينية المتوازية أثبتت نجاحها في تحسين كبس الصور الكسوري من خلال استخدام الثفرة

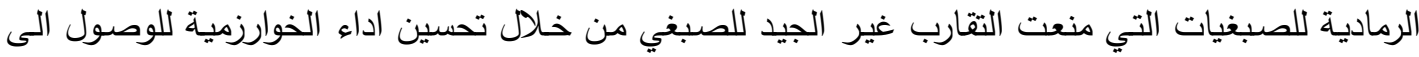

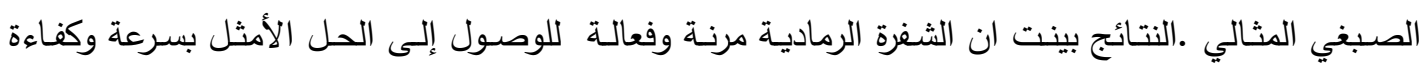

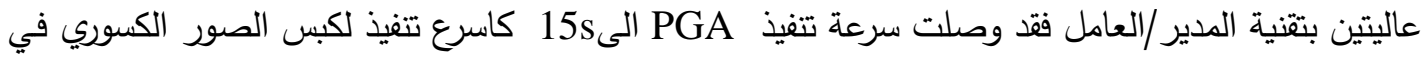
(6) حاسبات ,وان طريقة مجال البحث المستخدمة للكبس وفك الكبس ، واختيار معاملات الخوارزمية الجينية (الاختيار ،العبور والطفرة) كان له دور فعال في تحسين نسبة الكبس التي وصلت الى نوعية الصورة بعد فك الكبس التي وصلت الى مـا يقارب 34.81db ـ اوصسي باستخدام PGA في ايجاد الحلول لاي مشكلة اذا كان عامل السرعة مهما'لحلها، كما اوصي باستخدام هذه التقنية على الصور الملونة. 


\section{المصادر}

[1] Mingshui Li, Shanhu Ou and Heng Zhang: The new progress in research approach of fractal image compression. Journal of Engineering Graphics. 4(3),143-152(2004)

[2] A. Ben Jmaa, M. Ben Jemaa And Y. Ben Jemaa, "Optimization of fractal image compression with genetic algorithms", A. BEN JMAA is with National School of Engineering of Sfax,BPW,3038 Sfax ahmed.benjmaa@gmail.com,2006

[3] M. Mohamed ,"Optimization of fractal image compression based on kohonen neural networks", eedis Laboratory ,Engineering Faculty ,University of SBA ,mohamedmokht@yahoo.fr,2006

[4] X ,Lifeng, Z ,Liangbin,"A Study of Fractal Image Compression Based on an Improved Genetic Algorithim", Zhejiang Wanli University ,2007.

[5] Y.Chakrapani,K.Soundara," Genetic Algorithm Applied To Fractal Image Compression ", Dept. of Electronic and communications, J.N.T.U College of Engineering ,India,2009.

[6] P. Borovska,"Solving the Travelling Salesman Problem in Parallel by Genetic Algorithm on Multicomputer Cluster", Computer Systems Dept.,Technical University of Sofia,pborovska@tu-sofia.bg,2006.

[7] S.Tongchim,Prabhas ," Parallel Genetic Algorithm for Finite-State Machine Synthesis From Input/Output Sequences", Department of Computer Engineering,Chulalongkorn University Bangkok 10330, Thailand ,email g41stc@cp.eng.chula.ac.th,2000.

[8] M.Sheng,J.H. Jeng,"Schema genetic algorithm for Fractal Image Compression", Dept. of Electronic Engineering,N.S. Yet-Sen University,Taiwan ,2006.

[9] B.S.Sanjay,M.V. Kulkarni,"Fractal Image Compression Using Genetic Algorithim ",Advances in computer Engineering ACE,India , June 2010.

[10] Y.Chakrapani,K.Soundara," A Comparative Approach To Fractal Image Compression Using Genetic Algorithm and Simulated Annealing Technique ", Dept. of Electronic and communications,J.N.T.U College of Engineering ,India,2008.

[11] Y.Chakrapani,K.Soundara ,"Hybrid Genetic -Simulated Annealing Approach for Fractal Image Compression", Dept. of Electronic and communications J.N.T.U College of Engineering ,India,2008.

[12] Y.Chakrapani,K.Soundara ,"Implementation of Fractal Image Compression Hybrid Genetic -Neural Approach", Dept. of Electronic and communications ,J.N.T.U College of Engineering, India,Vol.7, No. 3,September 2009.

[13] Y.Chakrapani,K.Soundara ,"Adaptive Neuro-Fuzzy Inference System based Fractal Image Compression ", Dept. of Electronic and communications „J.N.T.U College of Engineering,India, Vol 2,No.1, November 2009.

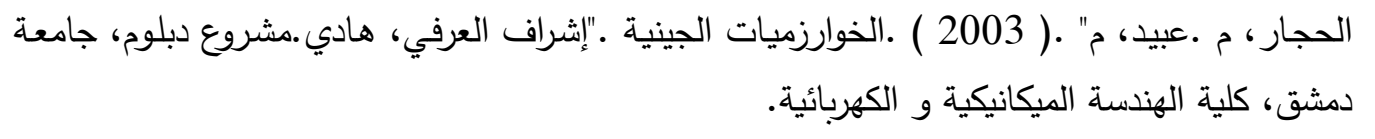

